\title{
Kombination optischer Rückstreusensorik und Single-Use-Technologie zur Prozessintensivierung von Zellkultur-Prozessen
}

\author{
M. Rotter ${ }^{1}$, T. Hanselmann ${ }^{2}$, L. Käßer ${ }^{1}$, J. Kneitz' , F. Eilts ${ }^{1}$, G. Dekevic ${ }^{1}$, G. Cremers², \\ D. Exner ${ }^{2}$, P. Czermak ${ }^{1,3}$, D. Salzig 1 \\ ${ }^{1}$ Institut für Bioverfahrenstechnik und Pharmazeutische Technologie - IBPT, \\ THM Gießen, Gießen, Deutschland \\ ${ }^{2}$ Exner Process Equipment GmbH, Ettlingen, Deutschland \\ ${ }^{3}$ FB08 Biologie und Chemie, Justus-Liebig Universität, Gießen, Deutschland
}

Kontakt: denise.salzig@lse.thm.de

\section{Einleitung}

Zur Herstellung von Biopharmazeutika werden prozessspezifische Kontrollstrategien genutzt, um eine hohe Kosteneffizienz zu erzielen und eine hohe Produktqualität zu garantieren (Quality-by-Design Ansatz, $Q b D$ ). Mittels der Bestimmung der entscheidenden Qualitätskriterien des Produktes und einer eingehenden Charakterisierung des Prozesses wird Prozessverständnis generiert und kritische Prozessparameter (CPPs) ermittelt. Dies ist die Basis einer engmaschigen Überwachung der Produktionsprozesse, was im Hinblick auf die umfassenden Regularien der guten Herstellungspraxis und die Zulassung des Produktes relevant ist.

Industrielle Zellkulturprozesse werden zunehmend in Single-Use-Reaktoren durchgeführt. Im Rahmen eines gemeinsamen Forschungsvorhabens der THM und der Exner Process Equipment $\mathrm{GmbH}$ wurde zur Überwachung und Steuerung von Zellkulturprozessen ein optischer Rückstreusensor mit innovativer Messoptik zur direkten Integration in Single-Use-Kultivierungssysteme entwickelt. Im Vergleich mit kommerziell erhältlichen Absorptionssensoren, liegt der Vorteil der Rückstreutechnologie vor allem in einem erweiterten linearen Messbereich. Damit sind Rückstreusensoren insbesondere für die Hochzelldichte in z.B. Perfusionsprozessen interessant. Die Entwicklung des Einweg-Sensors zielte auf einen hybriden Aufbau, bei welchem die Elektronik und Auswerteeinheit als autoklavierbare Multiple-Use Komponente, und Teile der optischen Einheit sowie der komplette Prozessanschluss als standardisierte Single-Use-Komponente ausgeführt wird. In einer breitangelegten Biokompatibilitätsstudie wurden geeignete Werkstoffe für die Fertigung des Sensors identifiziert.

Es wurden diverse Prototypen des Rückstreusensors bei der Entwicklung zweier Zellkulturprozesse getestet, und der entwickelte Sensor ermöglichte es das Zellwachstum als CPP in zwei innovativen Zellkulturprozessen inline zu überwachen.

Bei dem ersten Prozess handelte es sich um einen Perfusionsprozess zur Produktion antimikrobieller Peptide (AMPs). Für die Produktion von AMPs sind neue Prozessstrategien notwendig, da sich die Produktion in mikrobiellen Produktionssystemen aufgrund der antimikrobiellen Wirkung dieser Stoffe oft schwierig gestaltet. Daher wurde in diesem Prozess mit einer stabilen Insektenzelllinie gearbeitet und die Sensorsignale zur Regelung der Zellkonzentration während der Produktionsphase genutzt.

Der zweite Prozess dient der transienten Expression von virus-like-particles (VLPS) des Gelbfiebervirus in HEK 293 T-Zellen mit dem Ziel die VLPs für die passive Immunisierung verwenden zu können. Das Biomassemonitoring ist bei diesem Prozess vor allem in Bezug auf die zeitliche Abstimmung der beiden Schlüsselereignisse, Transfektion und Ernte der VLPS, von entscheidender Bedeutung.

\section{Methoden und Materialien}

Zellkultur und Kultivierungsbedingungen

Alle Zelllinien wurden durch spezifische Analysen regelmäßig auf deren Identität und Freiheit von Mykoplasmen geprüft. Die Bestimmung der Zellkonzentrationen und Vitalität erfolgte für jeden Versuch mittels Durchflusszytometrie und Trypanblaufärbung.

Eine rekombinante Sf-9 Zelllinie (Sf-9 TriEx ${ }^{\mathrm{TM}}$, Merck, Darmstadt, Germany) wurde für die Stammhaltung im 100-mL-Schikanekolben in Sf-900 ${ }^{\mathrm{TM}}$ ॥ (Thermo Fisher Scientific, Waltham, MA, USA) Insektenzellkulturmedium kultiviert. Die Zellen wurden zweimal pro Woche passagiert. Die Passagen wurden mit einer Vitalität $>90 \%$ und einer Zelldichte von $0,5^{*} 10^{6} \mathrm{Z} \mathrm{mL} \mathrm{mL}^{-1}$ angeimpft und im Brutschrank 
bei $28{ }^{\circ} \mathrm{C}$ und $80 \mathrm{rpm}$ kultiviert. Die Kryokonservierung erfolgte nach SOP der Zellbank.

Die an Suspensionskultivierung adaptiere HEK 293T SF Zelllinie (DSMZ no.: ACC 635) wurde in 100-mL-Schikanekolben mit BalanCD $\circledast$ oder FreeStyle ${ }^{\mathrm{TM}} 293$ Medium (Thermo Fisher Scientific, Waltham, MA, USA) kultiviert. Die Animpfkonzentration betrug $0,3-0,5^{\star} 10^{6} \mathrm{Z} \mathrm{mL}^{-1}$ bei einer Vitalität $>90 \%$. Die Kultivierung erfolgte bei $37^{\circ} \mathrm{C}, 8 \%(\mathrm{v} / \mathrm{v})$ $\mathrm{CO}_{2}$ und $100 \mathrm{rpm}$.

\section{Korrelation und Modellierung}

Auf Basis einfacher Schüttelkolbenkulturen wurden Modelllösungen mit definierten Zellkonzentrationen der Zelllinien Sf-9 und HEK 293T SF als biologisches Triplikat mit den Sensoren vermessen. Im Schüttelkolben durch Stofftransportlimitierungen unerreichbar hohe Zellkonzentrationen wurden durch Aufkonzentrierung mittels Zentrifugation erzielt. Die Aufnahme des Messignals erfolgte durch Eintauchen der Sensorprototypen in die Zellsuspension. Ausgehend vom ersten Ansatz wurden Verdünnungsreihen angefertigt und analog vermessen (Abbildung 1). Referenzwerte zu jedem Verdünnungsschritt wurden durch Zellzählung mittels Durchflusszytometrie, Trypanblaufärbung, sowie mit einer etablierten Trübungssonde nach dem Absorptionsprinzip aufgenommen. Die Modellierung der Korrelationsfunktionen erfolgte durch eine lineare oder quadratische Regression der partiellen kleinsten Quadrate (PLS).

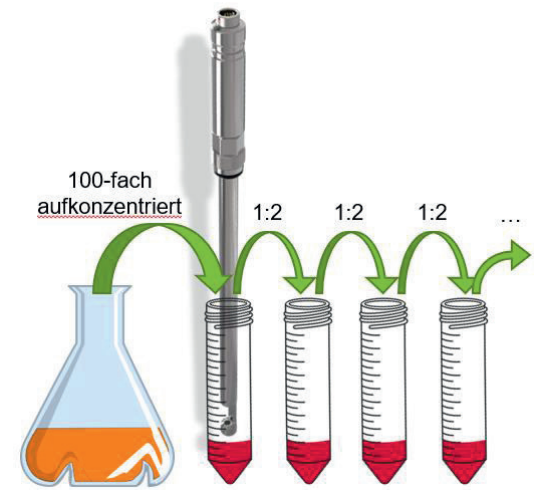

Abbildung 1: Schema der Verdünnungsreihe nach Aufkonzentrierung von Suspensionszellen aus Schüttelkolbenkulturen zur Aufnahme von Korrelationsfunktionen der Trübungssonden

Es wurden Trübungssonden eingesetzt die im Nahinfrarotbereich $(850 \mathrm{~nm})$ Licht emittieren und Streu- sowie Transmissionslicht detektieren. Die turbidimetrischen Sonden (Durchlichtsonden) detektieren die Abschwächung des eingestrahlten Lichts über eine festgelegte Distanz im Medium zwischen Detektor und LED. Demgegenüber wird von Rückstreusonden Streulicht detektiert, das in einem definierten Winkel auf die Photodioden des Detektors trifft. Einsatzgebiete und Unterschiede der Sondentypen sind in der Literatur umfassend beschrieben [1-4]. Eine Abbildung der verwendeten Multi-Use Sonden und Prototypen von Exner Process Equipment $\mathrm{GmbH}$ ist in Abbildung 2 dargestellt.

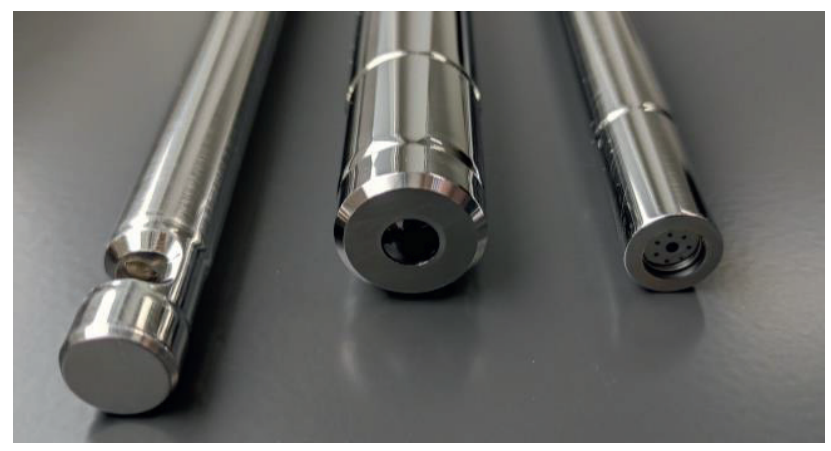

Abbildung 2: Eingesetzte Multiple-Use-Sonden aus Edelstahl für Korrelationsversuche und Bioreaktorkultivierungen, (links): Durchlichtsonde 2 mit $5 \mathrm{~mm}$ optischer Pfadlänge (OPL), (mittig): Rückstreusonde 1, (rechts): Rückstreusonde 3

Toxizitätsstudien und Materialauswahl

Potenzielle Kunststoffe für die Fertigung der Single-Use-Sonden wurden verschiedenen Zytotoxizitätsuntersuchen nach USP <87> und ISO 10993 unterzogen. Bei diesen wurden die Zelllinien L929 (DSMZ no.: ACC 2), die rekombinante Sf-9 Zelllinie, sowie adhärente HEK 293T Zellen verwendet. Eine Extraktion potenzieller Extractables und Leachables fand zellfrei unter Kultivierungsbedingungen für die verwendeten Zellkulturmedien RPMI 1640 $10 \%(\mathrm{v} / \mathrm{v}) \mathrm{FKS}+2 \mathrm{mM}$ L-GIn, Sf-900 TM II und DMEM $5 \%$ (v/v) FKS + 4 mM L-GIn in Anlehnung an ISO 10993-12 statt. Als Kontrollen für zytotoxische Einflüsse wurden 6-1,5\% (v/v) DMSO-Ansätze verwendet. Qualitative Untersuchungen der Zellmorphologien unter Extrakteinfluss der Kunststoffproben wurden mittels Cytation ${ }^{\mathrm{TM}} 3$ Digitalmikroskop (BioTek Instruments) durchgeführt. Nach ISO 10993-5 [5] und USP <87> [6] wurde der zytotoxische Einfluss klassifiziert. Wachstumskinetiken der drei Zelllinien über typische Kultivierungszeiten von 5 bis 7 Tagen unter Extrakteinfluss dienten der Beurteilung einer Wachstumshemmung. Mittels Durchflusszytometrie und Trypanblaufärbung wurden täglich Zellkonzentrationen sowie Vitalitäten bestimmt und die Glukose-, sowie Laktatkonzentration mittels BioSen C Line GP+ (EKF Diagnostics) detektiert. Eine weitere Untersuchung stellte nach 
ISO 10993-5 Annex C ein MTT-Assay mit den Zelllinien L-929 und HEK293T dar. Die Messung der Absorptionen und Berechnungen der relativen metabolischen Aktivität und Vitalität erfolgte im Plattenleser (BioTek Instruments).

\section{Kultivierungen im Bioreaktor}

Die Kultivierungen der Sf-9 Zelllinie erfolgten im $5 \mathrm{~L}$ Sartorius Bioreaktor. Der Rührkessel wurde mit einem Schrägblattrührer $\left(3 \times 45^{\circ}, d=74 \mathrm{~mm}, \mathrm{n}=\right.$ 100 rpm), einer PT100 Temperatursonde, einer $\mathrm{pH}$ Sonde, einer optischen Sauerstoffsonde, zweier OD-Sonden, sowie einer Impedanzsonde mit PreAmplifier ausgestattet. Die Baulänge der Sonden betrug $325 \mathrm{~mm}$. Die Vorbereitung und Durchführung der Kultivierung erfolgte analog zu Harnischfeger et al. [7].
Die HEK Zelllinie wurde in einem Bioreaktor mit $5 \mathrm{~L}$ Arbeitsvolumen kultiviert. In einer Vergleichskultivierung wurde die Rückstreusonde 3 und die Durchlichtsonde 2 im Prozessleitsystem je eines Kessels eingebunden.

\section{Ergebnisse und Diskussion}

In Abbildung 3 sind die Korrelationsdaten mit zugehörigen Korrelationsfunktionen der getesteten Sonden mit der rekombinanten Sf-9 Zelllinie dargestellt. Erwartungsgemäß zeigt sich eine lineare Korrelation für die Rückstreusonden 1 und 3 ( $B, C$ und D). Die Daten der Durchlichtsonde (A) weisen einen typischen asymptotischen Verlauf im oberen Konzentrationsbereich auf, während unter $20^{*} 10^{6} \mathrm{Z}$ $\mathrm{mL}^{-1}$ ein empfindlicher, linearer Zusammenhang
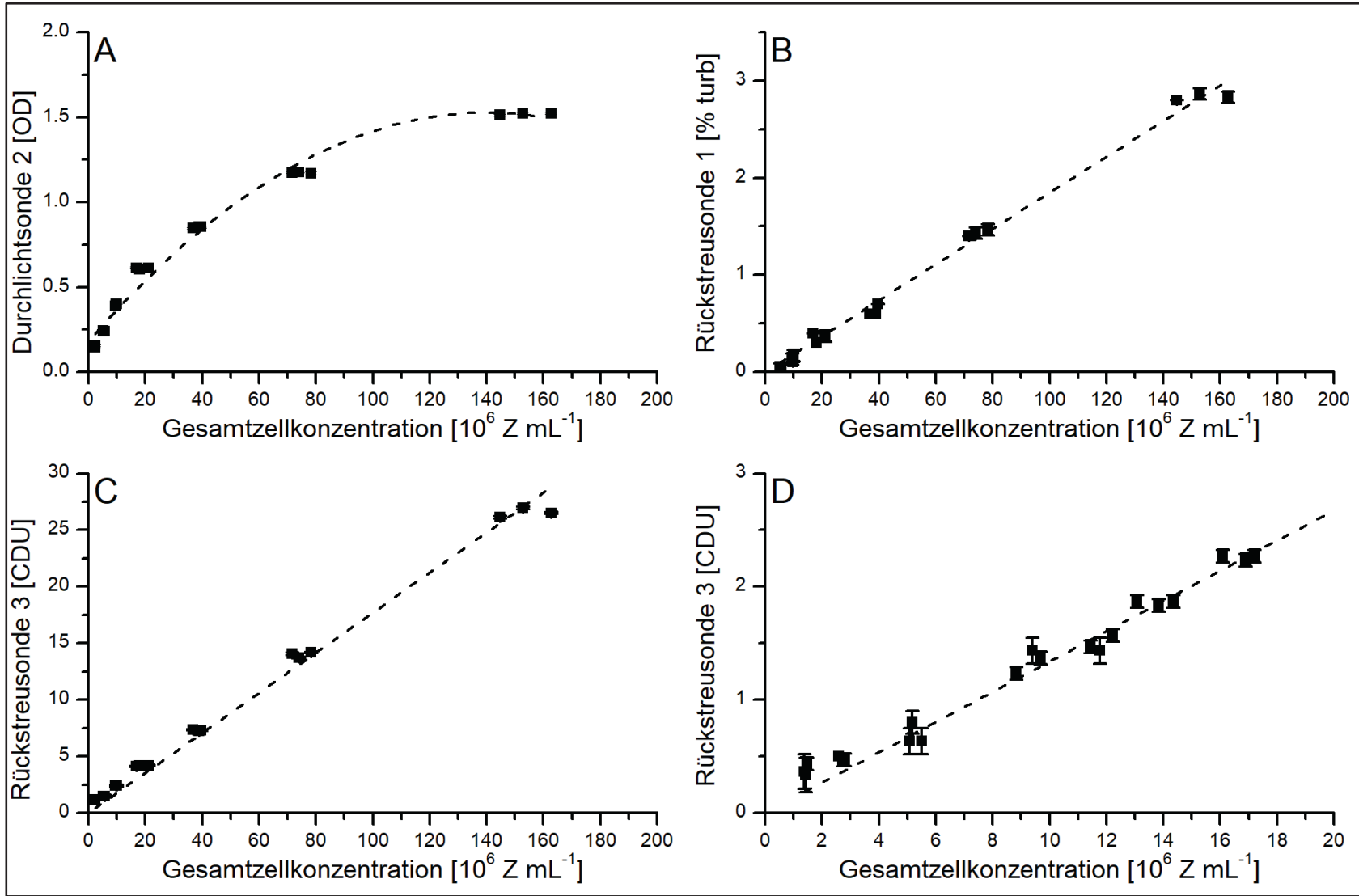

\begin{tabular}{lll}
\hline Typ & Korrelationsfunktion & $\mathbf{R}^{\mathbf{2}}[\%]$ \\
\hline $\mathbf{A}$ & $\mathbf{O D}_{\mathrm{A}}=-\mathbf{6 , 9} * \mathbf{1 0}^{-5} * \mathbf{c}_{\mathrm{G}}{ }^{\mathbf{2}}+\mathbf{1 , 9} * \mathbf{1 0}^{-\mathbf{2}} * \mathbf{c}_{\mathrm{G}}+\mathbf{0 , 1 8}$ & 98,2 \\
$\mathbf{B}$ & $\mathbf{C D U}_{\mathrm{B}}=\mathbf{1 , 8 8} * \mathbf{1 0}^{-2} * \mathbf{c}_{\mathrm{G}}-\mathbf{0 , 0 4}$ & 99,4 \\
$\mathbf{C}$ & $\mathbf{C D U}_{\mathrm{C}}=\mathbf{1 , 6 9} * \mathbf{1 0}^{-\mathbf{1}} * \mathbf{c}_{\mathrm{G}}+\mathbf{0 , 8 5}$ & 99,6 \\
$\mathbf{D}$ & $\mathbf{C D U}_{\mathrm{D}}=\mathbf{1}, \mathbf{2 3} * \mathbf{1 0}^{-\mathbf{1}} * \mathbf{c}_{\mathbf{G}}-\mathbf{0 , 1 4}$ & 98,2 \\
\hline
\end{tabular}

Abbildung 3: Korrelationsfunktionen unterschiedlicher Sensortypen und Messprinzipien für Sf-9 Zellen, Messignal [OD, \%turb, CDU] in Abhängigkeit der Gesamtzellkonzentration [106 $\mathrm{Z} \mathrm{mL}^{-1}$ ], Zellvitalität > 90 \% (A): Durchlichtsonde 2 (5 mm OPL), (B): Rückstreusonde 1, (C): Rückstreusonde 3, (D): Rückstreusonde 3 während Messung im unteren Messbereich, [CDU] als Customer defined unit (Einheit der Rückstreutechnik abweichend von Norm ISO 7027) 
besteht. Streu- und Verdeckungseffekte von hochkonzentrierten Partikeln erklären den asymptotischen Verlauf. Eine Anpassung der optischen Pfadlänge würde den linearen Messbereich verschieben $[8,9]$. In Zellkulturprozessen sind jedoch Konzentrationen ab etwa $50^{*} 10^{6} \mathrm{Z} \mathrm{mL}^{-1}$ meist nur durch Zellrückhalt und Perfusion möglich. Eine hohe Auflösung im unteren Messbereich ist daher für die weniger aufwendigen Prozessmodi wie Batch und Fed-Batch ausreichend. Im Gegensatz zur Rückstreusonde 1 ( $B$, Daten nicht gezeigt) wies Rückstreusonde 3 (D) auch im unteren Messbereich eine hohe Empfindlichkeit und Auflösung auf, die für eine Prozessintensivierung von Batch zu Perfusion erforderlich sind.

Analog zur Sf-9 Insektenzelllinie wurden Verdünnungsreihe mit HEK293T SF Zellen

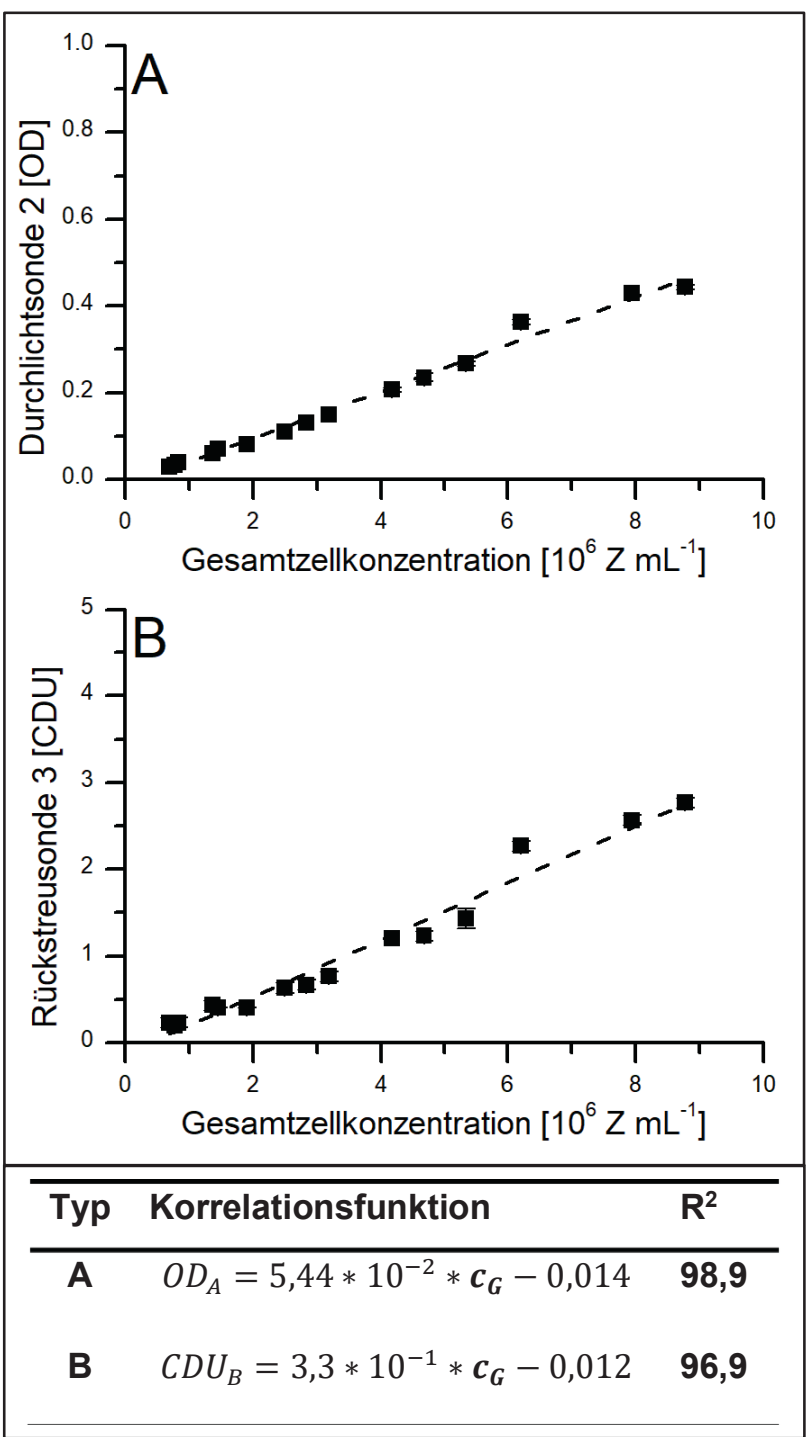

Abbildung 4: Korrelationsfunktionen für HEK293T SF Zellen, Messignal [OD, \%turb, CDU] in Abhängigkeit der Gesamtzellkonzentration [Z/mL], Zellviabilität > $90 \%$ (A): Durchlichtsonde 2, (B): Rückstreusonde 3 vermessen (Abbildung 4). HEK293T SF Zellen erreichten im Schüttelkolben geringere Zellkonzentrationen als Insektenzellen und mussten stärker aufkonzentriert werden. Die erwarteten Konzentrationen im Batch-Bioreaktor werden in der Literatur ebenfalls geringer angegeben: $3,5-7^{*} 10^{6} \mathrm{Z} \mathrm{mL}^{-1}$ für HEK $[10,11]$ und $7-16 \mathrm{Z} \mathrm{mL}^{-1}$ für $S f-9[12,13]$. Sowohl die Durchlichtsonde 2 als auch die Rückstreusonde 3 wiesen im relevanten Konzentrationsbereich eine lineare Korrelation auf. Die Sonden konnten nach diesen Vorversuchen beide im realen Prozess getestet werden.

\section{Toxizitätsstudien}

In Zytotoxizitätsstudien wurden unterschiedliche Polymere von mehreren Herstellern für die Fertigung der Single-Use Sonde getestet. In Abbildung 5 sind die Ergebnisse des MTT-Assays nach ISO 10993-5 Annex C dargestellt. Ein schädlicher Einfluss der Polymere würde die metabolische Aktivität der Zellen beeinflussen, wodurch sich eine geringe

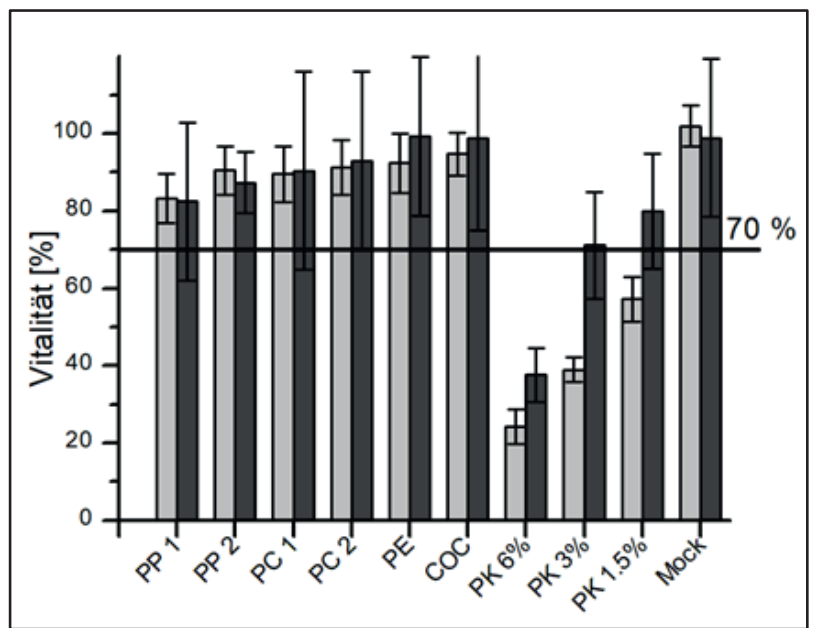

Abbildung 5: Vitalität der Zelllinien L929 $(n=5)$ (Hellgrau) und HEK293T ( $n=10$ ) (Dunkelgrau) bestimmt durch MTT-Assay für unterschiedliche Polymere mit Positivkontrolle DMSO (PK) und Mockkontrolle als frisches Medium

Vitalität im Assay ergibt. Eine Vitalität unter $70 \%$ ist ein Indikator für zytotoxische Einflüsse, erkennbar an der Positivkontrolle mit DMSO 6 \% (Abbildung 5).

Bei diesen Testungen wurde deutlich, dass Kunststoffe wie Polyethylen (PE), die nach USP Klasse VI Zertifizierung als biokompatibel eingestuft werden, unterschiedlich starke Einflüsse auf das Wachstum und den Metabolismus verschiedener Zelllinien zeigten. Des Weiteren erwiesen sich gleichartige Kunststoffe verschiedener Hersteller ebenfalls als unterschiedlich biokompatibel. Aus 
diesen Gründen ist für die Sicherheit und Produktqualität eine Testung potenziell vorgesehener Kunststoffe mit unterschiedlichen Zelllinien nötig. Eine Reduktion der Unsicherheit kann durch mindestens zwei orthogonale Testmethoden erfolgen. Anhand der zuvor beschriebenen Testmethoden konnten erfolgreich Polymere für die Fertigung unterschiedlicher Bauteile eingegrenzt werden.

\section{Bioreaktorkultivierung}

Kultivierungen im Bioreaktor dienten der Charakterisierung und dem Verständnisgewinn der beiden Prozesse. Das Monitoring der Zellkonzentration als $C P P$ ist neben den etablierten inline-Parametern wie $\mathrm{pH}$ und $\mathrm{O}_{2}$ unerlässlich für das Prozessverständnis. Die zuvor aufgenommenen Korrelationsdaten wurden nach Einbindung der Sonden im realen Prozess überprüft, um den Einfluss weiterer Störgrößen $z u$ beobachten. Im ersten Prozess wurde die Insektenzelllinie im Satzbetrieb zur Produktion von antimikrobiellen Peptiden kultiviert. Abbildung 6 zeigt die Daten der Satzkultivierung.

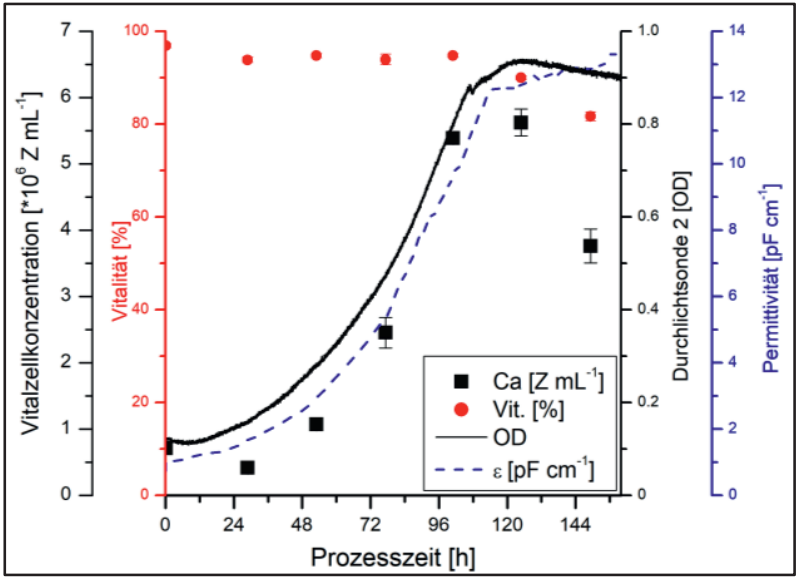

Abbildung 6: Batch-Kultivierung Sf-9, (schwarze Rechtecke): Offline-Zellkonzentration [ $\left.\mathrm{Z} \mathrm{mL}^{-1}\right]$, (schwarze Linie): Trübung der Durchlichtsonde 2 [OD], (blaue Linie): Online-Permittivität [pF cm$\left.{ }^{-1}\right]$ und (Rot): Vitalität [\%] während der Kultivierung über der Prozesszeit [h]

Sowohl das Trübungssignal als auch die Daten der Permittivitätsmessung folgen den Offline-Referenzwerten der Zellzählung und spiegeln das Wachstum der Zellen wider. Ab Tag 5 trennten sich die Signale der Trübung und Permittivität. Dies ist mit dem Übergang der Zellen in die stationäre Wachstumsphase zu erklären. Das Anschwellen von Insektenzellen am Ende einer Satzkultivierung führt zu einer Erhöhung des Zellvolumens, welches gut mit der gemessenen Permittivität korreliert. Demgegenüber wird die Trübung durch die Gesamtzellkonzentration bestimmt, die während des Übergangs zur stationären Wachstumsphase konstant bleibt und in der nachfolgenden Absterbephase durch Zelllyse sinkt. Die in der ersten Kultivierung eingesetzte Durchlichtsonde bestätigt die in den Korrelationsversuchen ermittelte Empfindlichkeit im relevanten Konzentrationsbereich von 0,5 bis $7^{*} 10^{6} \mathrm{Z} \mathrm{mL}^{-1}$. Die Verwendung von Nahinfrarot-Trübungssonden (NIR) wird in der Literatur für unterschiedliche Zellkulturprozesse einschließlich Insektenzellkultur beschrieben $[1,8,14]$. Die Begasung durch den verwendeten Ringsparger zeigte keinen erheblichen Einfluss auf die Stabilität des Messsignals. Im Gegensatz zur dielektrischen Spektroskopie kann die Trübungsmessung durch jegliche Partikel und dispergierte Blasen aufgrund von Streueffekten beeinflusst werden $[9,15]$. Eine geeignete Dämpfung des Messwertes wurde durch die Bildung des gleitenden Durchschnitts $(n=5)$ mit dynamischem Fenster durchgeführt. Des Weiteren kann Rauschen, das z.B. durch die Interaktion einer Gasblase am Messfenster durch digitale Unterdrückung herausgefiltert werden. Sowohl die Parameter der Dämpfung als auch der Rauschunterdrückung sind abhängig von dem eingesetzten Kultivierungssystem und vorhandenen Störgrößen und erfordern eine Parametrierung unter realen Prozessbedingungen. So bedarf der Einsatz der Trübungssonden in mikrobiellen Fermentationen aufgrund der hohen Anzahl kleiner dispergierter Blasen eine erheblich höhere Glättung des Messwertes, die wiederum zu Verzögerungen im Ansprechverhalten führt. (Daten nicht gezeigt)

In einer Kultivierung von HEK-Zellen wurde ein Prototyp der Rückstreusonde 3 mit erhöhter Auflösung im unteren Konzentrationsbereich erfolgreich eingesetzt. In Abbildung 7 sind die Kultivierungsdaten einer HEK-Zelllinie dargestellt.

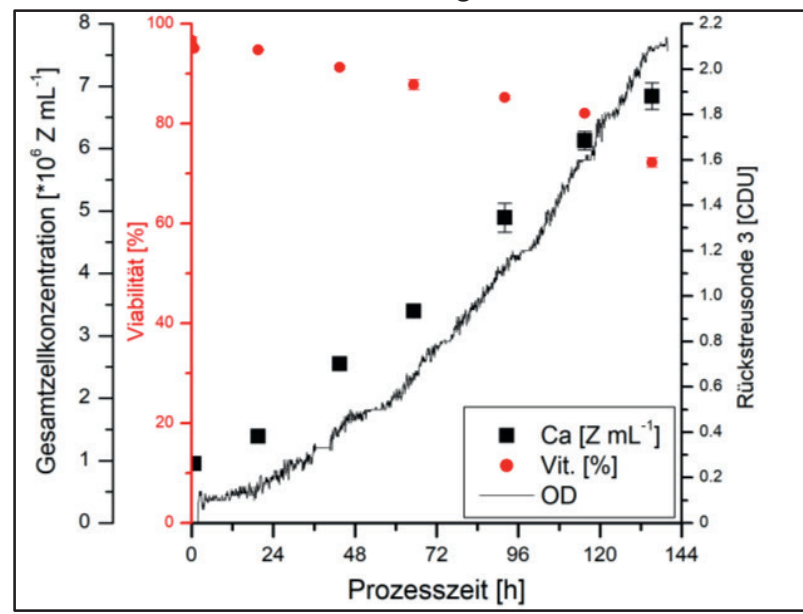

Abbildung 7: Fed-Batch-Kultivierung von HEK-Zellen in 5 L Kulturmedium, (Schwarz): Offline-Zellkonzentration [Z mL-1 ], (Rot): Vitalität [\%], (schwarze Linie): Trübung der Rückstreusonde 3 [CDU] während der Kultivierung über der Prozesszeit [h]. 
Im untersuchten Konzentrationsbereich wiesen die Trübungsdaten ein Rauschen auf. Das Trübungssignal wurde daher im Anschluss an die Kultivierung mittels eines gleitenden Durchschnitts $(n=10)$ geglättet. Zur Implementierung der Sonde für die zeitliche Abstimmung von Schlüsselereignissen wie Transfektion und Ernte werden derzeit weitere Reaktorkultivierungen durchgeführt. Mittels kombinierter PAT-Technologie wie dielektrischer Spektroskopie und Turbidimetrie wird in etablierten Kultivierungssystemen Prozessverständnis generiert, das den Transfer des Prozesses in Single-use Systeme ermöglicht.

\section{Literatur}

1. Käßer L, Zitzmann J, Grein T, Weidner T, Salzig D, Czermak P. Turbidimetry and Dielectric Spectroscopy as Process Analytical Technologies for Mammalian and Insect Cell Cultures. In: $\mathrm{GmbH}$ T, editor. Balearen. 2014th ed. Bielefeld: Delius Klasing; 2015. p. 335-364. doi:10.1007/978-1-0716-0191-4_20.

2. Wu P, Ozturk SS, Blackie JD, Thrift JC, Figueroa C, Naveh D. Evaluation and applications of optical cell density probes in mammalian cell bioreactors. Biotechnology and Bioengineering. 1995;45:495-502.

doi:10.1002/bit.260450606.

3. Junker BH, Reddy J, Gbewonyo K, Greasham R. On-line and in-situ monitoring technology for cell density measurement in microbial and animal cell cultures. Bioprocess and Biosystems Engineering. 1994;10:195-207. doi:10.1007/BF00369530.

4. Konstantinov K, Chuppa S, Sajan E, Tsai Y, Yoon S, Golini F. Real-time biomass-concentration monitoring in animal-cell cultures. Trends in Biotechnology. 1994;12:32433. doi:10.1016/0167-7799(94)90049-3.

5. International Organization for Standardization. DIN EN ISO 10993-5: 2009-06-01 Biological evaluation of medical devices - Part 5: Tests for in vitro cytotoxicity.

6. The United States Pharmacopeial Convention. <87> Biological Reactivity Tests, In Vitro.

7. Harnischfeger J, Käßer L, Zitzmann J, Salzig D, Czermak P. Bioreactor-Based Antigen Production Process Using the Baculovirus Expression Vector System. In: Pfeifer BA, Hill A, editors. Vaccine Delivery Technology: Methods and Protocols. 1st ed. New York, NY: Springer US; Imprint Humana; 2021. p. 95-118. doi:10.1007/978-1-0716-07954_8.

8. Cervera AE, Petersen N, Lantz AE, Larsen A, Gernaey $\mathrm{KV}$. Application of near-infrared spectroscopy for monitoring and control of cell culture and fermentation. Biotechnology Progress. 2009;25:1561-81. doi:10.1002/btpr.280.

9. Biechele P, Busse C, Solle D, Scheper T, Reardon K. Sensor systems for bioprocess monitoring. Eng. Life Sci. 2015;15:469-88. doi:10.1002/elsc.201500014.

10. Liste-Calleja L, Lecina M, Cairó JJ. HEK293 cell culture media study: increasing cell density for different bioprocess applications. BMC Proc 2013. doi:10.1186/17536561-7-S6-P51.

11. Liste-Calleja L, López-Repullo J, Lecina M, Cairó JJ. Preliminary studies of cell culture strategies for bioprocess development based on HEK293 cells. BMC Proc 2013. doi:10.1186/1753-6561-7-S6-P52.

12. Cao W, Cao H, Yi X, Zhuang Y. Development of a simple and high-yielding fed-batch process for the production of porcine circovirus type 2 virus-like particle subunit vaccine. AMB Expr. 2019;9:164. doi:10.1186/s13568-0190880-8.

13. Gorenflo VM, Pfeifer TA, Lesnicki G, Kwan EM, Grigliatti TA, Kilburn DG, Piret JM. Production of a self-activating CBM-factor $\mathrm{X}$ fusion protein in a stable transformed Sf9 insect cell line using high cell density perfusion culture. Cytotechnology. 2004;44:93-102. doi:10.1007/s10616005-0703-4.

14. Qiu J, Arnold MA, Murhammer DW. On-line near infrared bioreactor monitoring of cell density and concentrations of glucose and lactate during insect cell cultivation. Journal of Biotechnology. 2014;173:106-11. doi:10.1016/j.jbiotec.2014.01.009

15. Zitzmann J, Weidner T, Eichner G, Salzig D, Czermak P. Dielectric Spectroscopy and Optical Density Measurement for the Online Monitoring and Control of Recombinant Protein Production in Stably Transformed Drosophila melanogaster S2 Cells. Sensors. 2018;18:900. doi:10.3390/s 18030900

\section{Danksagung}

Wir danken Ralf Amann (Department of Immunology, University of Tuebingen, Tuebingen, Germany + PRiME Vector Technologies, Tuebingen, Germany) und Felix Pagallies (Department of Immunology, University of Tuebingen, Tuebingen, Germany) für die Bereitstellung der Zelllinie und Michael W Wolff (Institut für Bioverfahrenstechnik und Pharmazeutische Technologie \& Fraunhofer-Institut für Molekularbiologie und Angewandte Oekologie IME, Gießen, Deutschland) für die Supervision der HEK-Kultivierung. 